\title{
Modeling of Protein Signaling Networks in Clinical Proteomics
}

\author{
D.H. Geho, E.F. Petricoin, L.A. Liotta, And R.P. Araujo \\ Center for Applied Proteomics and Molecular Medicine, Department of Molecular and Microbiology, \\ George Mason University, Manassas, Virginia 20110
}

\begin{abstract}
Molecular interactions that underlie pathophysiological states are being elucidated using techniques that profile proteomic endpoints in cellular systems. Within the field of cancer research, protein interaction networks play pivotal roles in the establishment and maintenance of the hallmarks of malignancy, including cell division, invasion, and migration. Multiple complementary tools enable a multifaceted view of how signal protein pathway alterations contribute to pathophysiological states. One pivotal technique is signal pathway profiling of patient tissue specimens. This microanalysis technology provides a proteomic snapshot at one point in time of cells directly procured from the native context of a tumor microenvironment. To study the adaptive patterns of signal pathway events over time, before and after experimental therapy, it is necessary to obtain biopsies from patients before, during, and after therapy. A complementary approach is the profiling of cultured cell lines with and without treatment. Cultured cell models provide the opportunity to study short-term signal changes occurring over minutes to hours. Through this type of system, the effects of particular pharmacological agents may be used to test the effects of signal pathway inhibition or activation on multiple endpoints within a pathway. The complexity of the data generated has necessitated the development of mathematical models for optimal interpretation of interrelated signaling pathways. In combination, clinical proteomic biopsy profiling, tissue culture proteomic profiling, and mathematical modeling synergistically enable a deeper understanding of how protein associations lead to disease states and present new insights into the design of therapeutic regimens.
\end{abstract}

The drive to create personalized approaches for medical care is underpinned by two attractive, interrelated endpoints: the elucidation of a molecular description for a patient's disease along with an effective and minimally toxic therapeutic regimen. Because of our currently limited understanding of how commonly used therapeutics effect their functions, patients often receive a battery of agents in a cocktail or in succession. Unfortunately, the conventional therapies are successful for only a subset of patients, and responses are often temporary. Moreover, selected therapies may be ineffective for a given patient's disease, thereby exposing the patient to the unwanted symptomatic or pathological side effects caused by the ineffective drug. One approach toward the improvement of therapeutic outcome shifts the therapeutic targets beyond individual molecules to entire networks inside and outside the cell in the context of the tissue microenvironment. Characterization of such molecular network defects that underlie disease states such as cancer will reveal insights into pathophysiology and provide molecular targets for intervention.

A remarkably varied palette of biomolecules, including nucleic acids, proteins, lipids, and carbohydrates, coalesce to form a molecular system in an organism. Although significant effort has been invested in cataloging and studying the nucleic acid content of biological systems, an incomplete picture emerges when only genetic material is used to study a complex system such as a cell or tissue microenvironment. Although DNA is an information archive, proteins perform pivotal structural, synthetic, signaling and recognition functions. Of particular interest in cancer, protein signaling pathways consist of transient networks of proteins that come together and dis- perse in a dynamic manner (Hunter 2000). In these signaling cascades, one protein binds to another and, in so doing, confers a posttranslational modification such as phosphorylation or cleavage. These subtle molecular changes are excluded from analysis by a genomics-only approach to molecular profiling.

For these reasons, molecular, personalized diagnostics for patients will require high-throughput profiling of the proteomic content within cells of interest. Clinical proteomics is a rapidly evolving discipline that has arisen out of this pressing need to generate molecular portraits of disease. Protein microarrays enable low-abundance proteins to be studied in a reproducible, high-throughput system (MacBeath and Schreiber 2000; Zhu and Snyder 2001; MacBeath 2002; Liotta et al. 2003). With this approach, known protein targets are probed using isoformspecific antibodies. The relative abundance of key proteins can be compared across specimens, providing a method for assessing the activity levels of key nodes within signaling protein pathways.

Because it is a fundamental means of personalizing diagnostics, profiling of actual patient tissues is a central goal of clinical proteomics. As a complementary approach for studying cellular signaling pathways in a rapid, real-time format, proteins extracted from cell culture assays are also being incorporated into protein microarrays. Mathematical modeling is required to organize the large volumes of information emanating from microarray studies, yielding insight into how signaling networks interact in a disease state. These three approaches, taken together, are offering new insights into the pathophysiology and treatment of diseases such as cancer. 


\section{PROTEOMIC PROFILING OF PATIENT BIOPSIES}

Technological challenges have, until recently, precluded systematic studying of the proteomic information with a patient's tissue specimen. One inherent difficulty in profiling the proteomic content of a tissue is that much important information resides in a population of lowabundance molecules. Whereas nucleic acid research is facilitated by polymerase chain reaction technologies, no comparable intrinsic amplification mechanism has yet been discovered for proteomic studies. Therefore, detection platforms with a high degree of sensitivity are required to glean an accurate picture of a tissue's proteomic profile.

\section{HANDLING OF BIOPSY MATERIAL FOR PROTEOMIC ANALYSIS}

For the promise of molecular medicine to be realized fully, the information contained within the biopsy must be translated into a molecular format. Thus, the process of molecular retrieval is central to the quality of information that is generated. When a biopsy is procured, the equivalent of a snapshot of a patient's disease process is obtained. As such, how it is handled determines the amount and quality of information that is derived from the procedure. For clinical proteomics research, immediately after a biopsy is obtained it must be snap-frozen in an embedding medium and then kept at $-80^{\circ} \mathrm{C}$ for long-term storage in order to preserve labile molecules in the sample. For further study, a thin frozen section is made of the tissue. On morphological inspection of the cells within a patient's biopsy specimen, it is apparent that numerous cell types are found within a tissue microenvironment. For example, in cancer specimens, there are not only tumor cells, but also the surrounding cells that contribute to the pathophysiological state. These cells may include nerves, blood vessels, fibroblasts, smooth muscle, lymphocytes, other inflammatory cells, and extravasated red blood cells (Liotta and Kohn 2001). Laser capture microdissection, however, provides a means for isolating a pure cell population from such a heterogeneous cellular environment (Emmert-Buck et al. 1996). Once purified cells from the biopsy are isolated, the protein content of the cells is extracted and can be utilized in profiling technologies such as the protein microarray.

\section{PROTEIN MICROARRAY FORMATS}

Two formats for protein microarrays have been implemented (Liotta et al. 2003). One method uses an antibody capture strategy and is called the forward phase array. The capture antibody for an analyte is immobilized onto a substrate, while a second antibody is used to detect a distinct epitope on the same analyte. The requirement of two antibodies in a forward phase array is a marked limitation of this approach, because specific antibodies for two distinct epitopes can be difficult, either because of limited antibody reagents or, alternatively, the analyte may be very small. A more efficient system requires the use of only one antibody per analyte. A format that enables this type of array is called the reverse phase microarray (RPMA). With the RPMA, proteins extracted from cells purified out of human tissues by laser capture microdissection are arrayed directly onto a substrate such as nitrocellulose. The proteins are spotted onto an array using an arraying device, similar to those utilized in genomic array systems (Fig. 1). The heterogeneous proteins from a cellular extract are arrayed directly onto such a

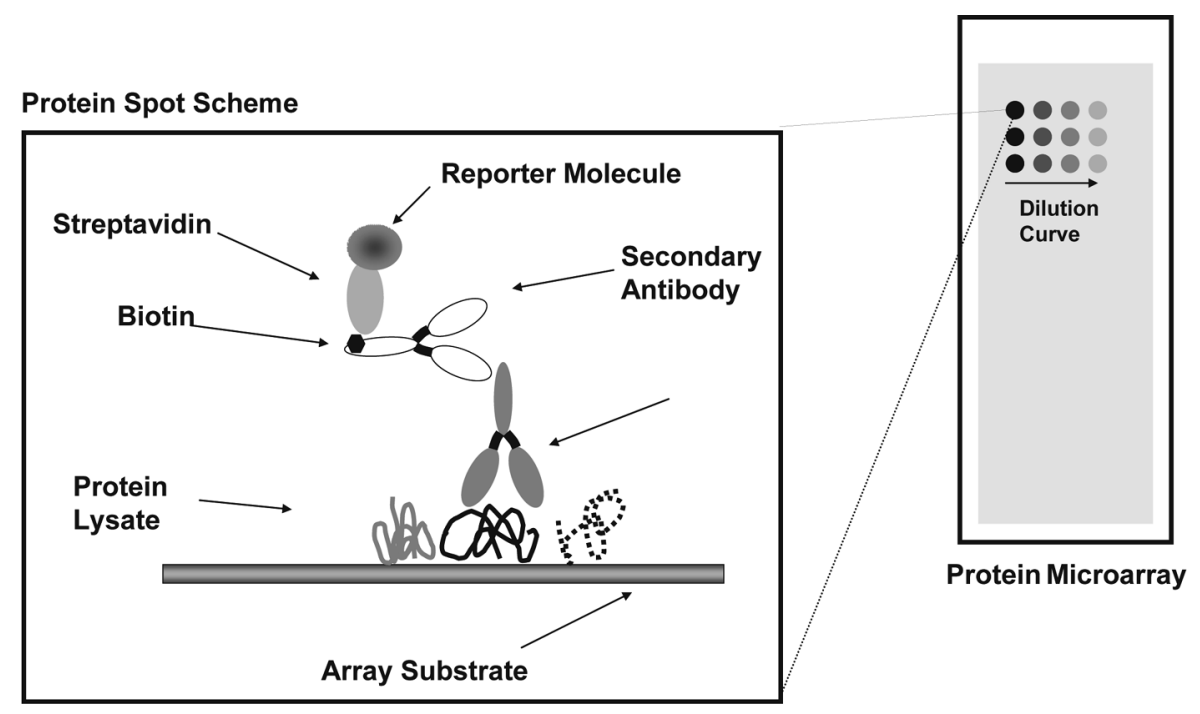

Figure 1. Standard reverse phase microarray. Protein analytes harvested from a purified cell population, obtained either through laser capture microdissection or cells from culture, are treated to extract the proteins contained within. The proteins, also called analytes, are arrayed onto a substrate such as nitrocellulose. Unbound sites on the nitrocellulose are then blocked. The immobilized analytes are probed with an antibody specific for protein endpoint of interest. These can be endpoints such as phosphorylated signaling proteins. The primary antibody is then detected using a secondary antibody specific for the isoform of the primary antibody. This secondary antibody is labeled with a biotin group. The biotin is detected using streptavidin linked to a reporter molecule, such as a quantum dot or horseradish peroxidase. 
substrate in a series of dilutions. In the standard RPMA format, a primary antibody specific for a known target protein, such as a phosphorylated isoform of a signaling protein, is incubated on the protein spot. Over a hundred antibodies validated by western blots are currently used to probe targeted analytes within protein extracts. These antibodies provide a means to study growth-regulatory, apoptotic, and cytoskeleton-linked pathways. Bound primary antibody is then detected by a secondary antibody that is biotinylated. As with immunohistochemistry, the secondary antibody is bound by streptavidin linked to a catalyst reporter molecule such as horseradish peroxidase (HRP). Because the proteins of interest are of low abundance, a biotinyl tyramide signal amplification system is used, which significantly enhances the sensitivity of the RPMA (King et al. 1997). After biotinyl tyramide groups are deposited, streptavidin-HRP is applied again followed by enzymatic deposition of a chromagen such as diaminobenzidene (DAB). This sensitive system can detect protein levels approaching attogram amounts. It is estimated that in the range of 1,000-5,000 molecules per spot can be detected using this system. Furthermore, utilization of patient material is maximized using this system, as 10,000 procured human cells allow the production of up to 100 RPMAs.

In an effort to increase the versatility of RPMAs, streptavidin-conjugated quantum dots have been used as an alternative reporter agent for RPMA (Geho et al. 2005). Through this study, it was determined that a pegylated form of the streptavidin-quantum dot bioconjugate was required, as nonpegylated reagent bound nonspecifically to arrayed protein spots. One benefit of this reporter technique is that signals can be detected without amplification when sensitive hyperspectral imaging is employed (Schultz et al. 2001; Huebschman et al. 2002). Another benefit of the quantum dots is their potential to be used in a multiplexed format, since they have narrow emission spectra. The development of a multiplexed format will enable more protein targets, or endpoints, to be detected on a single array surface. Each antibody probe could be linked to a particular type of quantum dot, yielding a characteristic emission spectrum. A further benefit of the quantum dot technology is that these fluorophores are resistant to photobleaching, unlike organic fluorophores, and can be imaged months after the original excitation.

As an additional strategy for increasing the amount of antibody probes that can be used with each slide, a sector array format has also been developed (Espina et al. 2004). This approach requires the engineering of a reservoir system and matching glass slide with distinct nitrocellulose pads, or sectors. With this type of array, multiple sectors are present on a single array surface, each of which may be incubated with a unique reaction condition. The sector array format significantly increases the amount of information that may be gleaned from a single array slide.

The RPMA allows a target analyte to be compared across various clinical specimens on the same array. This format enables low-abundance endpoints such as phosphorylated, or activated, signaling proteins to be detected among specimens. To perform comparisons, a reference standard can be included in the array (Sheehan et al.
2005). One type of reference standard is a mixture of known analytes such as synthetically produced peptides, with known concentrations. If a source such as peptides is used as the reference standard, the standard is renewable and chemically defined, which enhances its reproducibility. With a reference standard included on each array, the relative abundance of important signaling molecules can be assessed across specimens.

Of particular interest using these techniques has been the profiling of known signaling proteins. An early study using RPMA format demonstrated that pro-survival signaling pathways were key to the development of a cancer invasion front in prostate cancer (Paweletz et al. 2001). Signaling protein studies have been extended to other disease states as well, such as lymphoma and ovarian cancer (Wulfkuhle et al. 2003; Zha et al. 2004; Sheehan et al. 2005). Bioinformatic clustering analyses enable molecular profiles to be grouped according to similarities in protein populations. The RPMA format enables numerous distinct protein endpoints within cells to be measured and compared across disease types and, within disease types, across the spectrum of disease from in situ disease to advanced metastatic disease. Furthermore, the effects of therapeutic interventions on defined molecular endpoints can be monitored using this type of diagnostic system.

\section{PROTEOMIC PROFILING OF TREATED CELLS IN CULTURE}

The previously described format employed patient biopsy specimens as the source of the proteomic material for profiling (see Fig. 2). Previous work demonstrated that a significant gulf stands between cells grown in culture and those extracted from disease tissue (Ornstein et

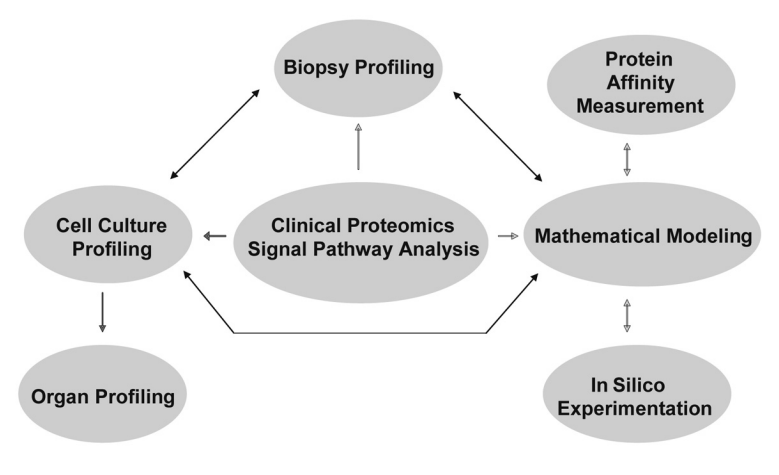

Figure 2. Proteomic signal pathway profiling. Signal pathway profiling is emerging as a multifaceted discipline employing distinct yet complementary tools. Proteomic profiling of biopsy specimens provides unique access to the molecular content of patient material. This enables a personalized molecular portrait to be fashioned. Tissue culture, or higher-order cultures, provide a real-time laboratory for measuring alterations in signaling pathways following treatment with pharmacological agents. Mathematical modeling studies provide a means for understanding complex signaling networks. As models grow in sophistication, theoretical in silico experimentation will provide a means of stratifying molecular targets as potential therapeutic targets. 
al. 2000). It is essential, when providing a molecular diagnosis for a patient, to study the cells within their native microenvironment using laser capture microdissection. However, this provides a "snapshot" of the tissue's proteomic activity, frozen in time. To study the kinetics of signaling pathways, an alternative and complementary system is required. Cell culture systems that possess the signaling molecule pathways of interest provide a unique means to measure the response of the pathways to pharmacological perturbation. Using similar protein extraction techniques and RPMA technology, the temporal variations in protein activities in particular pathways can be monitored in a realtime framework. Treatment of cells in culture with defined chemotherapeutic agents also represents a technique for elucidating the mechanism of drug activities. In one case study, a combination therapy comprising carboxyamidotriazole (a voltage-independent calcium channel inhibitor) and a celecoxib analog was shown to have a supra-additive growth inhibition in cell culture conditions (Winters et al. 2005). The RPMA format elucidated the mechanism of the cytotoxic activity; namely, with these two agents the cytotoxicity is effected through the suppression of proliferation and the initiation of apoptosis.

Three-dimensional spatial and temporal fluctuations of heterotypic and homotypic cell physiological functions acting within the architectural setting of an intact tissue or organ affect the activity levels of signaling molecules. To more closely approximate the cellular and molecular complexity of a tissue microenvironment in culture systems, organs or organ fragments may in the future be maintained in tissue reactors for cell signaling assays. Following treatment of the organized tissue with defined agents, the tissue could then be frozen and microdissected, in a manner similar to patient biopsies described above. This scenario will provide a more complex setting for studying cellular signaling pathways. Cells maintained within an organ or tissue context provide a more realistic portrait of molecular events than isolated tissue culture cells because of the presence of three-dimensional architectural elements, comprising stromal cells and extracellular matrix, which surround a cell in such a tissue context. These three-dimensional elements necessarily affect cellular signaling and must be taken into account in order to build more comprehensive models of cellular signaling events.

\section{MATHEMATICAL MODELING IN CLINICAL PROTEOMICS}

Notwithstanding the potential of RPMA technology to furnish an array of information on a given tumor's proteomic fingerprint, an important consideration from a translational standpoint is how to organize this information into a coherent picture of the dysfunction of the signal transduction networks, and to use this understanding to develop an effective therapeutic regimen to eradicate the tumor. Indeed, even with the inchoate understanding of intracellular biochemical control prevailing today, it is recognized that a cell has very subtle and complex techniques at its disposal to process biochemical signals (Tyson et al. 2003). Even the simple phosphorylation cycle formed by a kinase and an opposing phosphatase - a fundamental building block in any network of protein-protein interactions - exhibits important control characteristics through its ability to create an ultrasensitive "switch-like" response to an upstream signal under certain conditions (Goldbeter and Koshland 1981). Moreover, this simple signaling motif generally occurs in a layered structure to form a so-called "kinase cascade," which is itself interconnected with other cascades, and interlaced with manifold control interactions such as feedback and feed-forward loops (Fig. 3). Operating in concert with these mechanisms are still other features such as redundancy and spatiotemporal compartmentalization, which further complicate the processing of biochemical signals.

Thus, in view of the daunting complexity of signaling networks, both topologically and functionally, the effects of a particular therapeutic intervention on particular parts of the cell signaling network, and on the cell's behavior as a whole, may be recondite and unintuitive. Furthermore, the difficulties in understanding intracellular responses to therapeutic regimens are magnified considerably in the context of "combination therapies" (Araujo et al. 2004, 2005) — where multiple nodes in the cell's signaling net-

$$
\begin{aligned}
\mathrm{A} \\
\mathrm{B} \\
\frac{d[R]}{d t}=-k_{1}[S][R]+k_{-1}[P 1] \\
\frac{d[P 1]}{d t}=k_{1}[S][R]-k_{-1}[P 1]-k_{2}[P 1]+k_{-2}[P 2] \\
\frac{d[P 2]}{d t}=k_{2}[P 1]-k_{-2}[P 2]-k_{3}[P 2]+k_{-3}[P 3]-f_{5}[P 5] \\
\frac{d[P 3]}{d t}=k_{3}[P 2]-k_{-3}[P 3]-k_{4}[P 3]+k_{-4}[P 4] \\
\frac{d[P 4]}{d t}=k_{4}[P 3]-k_{-4}[P 4]-k_{5}[P 4]+k_{-5}[P 5] \\
\frac{d[P 5]}{d t}=k_{5}[P 4]-k_{-5}[P 5]-k_{6}[P 5]+k_{-6}[P 6] \\
\frac{d[P 6]}{d t}=k_{6}[P 5]-k_{-6}[P 6]-k_{7}[P 6]
\end{aligned}
$$

Figure 3. Mathematical modeling of a signaling molecule cascade with feedback inhibition. A simplified model of a hypothetical six-node kinase cascade (adapted from Araujo et al. 2004). (A) An extracellular stimulus (S) binds to a free receptor $(\mathrm{R})$ on the cell membrane, leading to the phosphorylation of the first protein $(\mathrm{P} 1)$ in the cascade. Each protein then phosphorylates the next protein downstream: P1 phosphorylates P2, P2 phosphorylates P3, and so on. Proteins may also be dephosphorylated by phosphatases, giving rise to a reverse reaction between a phosphorylated protein and the preceding upstream protein. Moreover, any given phosphorylated protein may promote or inhibit the phosphorylation of upstream or downstream proteins. A negative feedback loop is shown with a dashed line here as an example, with protein P5 inhibiting the phosphorylation of the upstream protein P2. (B) Modeling equations corresponding to the simple network in $A$. Note that these equations are based on the assumption of mass action kinetics, which neglects some of the control subtleties afforded by Michaelis-Menten kinetics. The basic model may be supplemented by feedback loops by making simple additions to the modeling equations. In the example given here, the negative feedback loop shown with a dashed line in $A$ is accounted for by the extra term shown with a dashed line surrounding it in B. (Adapted from Araujo et al. 2004.) 
work are targeted simultaneously — owing to the nonadditive effects of the individual agents. Early modeling studies on even the very simplest mathematical representations of cell signaling networks - with minimal feedback control or ultrasensitive characteristics - reveal that inhibiting a number of serially connected processes in a signaling cascade can produce a supra-additive (synergistic) attenuation of downstream signals in comparison with targeting the same processes individually (Araujo et al. 2005).

For this reason, the development of a successful patient-tailored therapy based on detailed RPMA-obtained information is dependent on the concomitant development of mechanistic mathematical models to bridge the divide between the molecular portrait of an individual patient's aberrant signaling network and an effective treatment. In this manner, the most effective complement of therapeutic targets may be determined, and an in silico drug trial conducted.

\section{FROM PROMISE TO REALITY: THE CHALLENGES AHEAD FOR INDIVIDUALIZED MOLECULAR MEDICINE}

\section{Reconciling Static and Dynamic Kinetic Signatures in Signaling Pathways}

The union of RPMA analysis of an individual patient's tissue biopsy with mathematical paradigms which interpret this information and point the way to the corresponding most effective therapy promises to be a fecund one, with the potential to revolutionize the treatment of disease. Nevertheless, for this concept to become a reality, the complexity of its implementation must be appreciated, and a clear plan for overcoming current challenges must be formulated.

For individualized molecular-targeted medicine to realize its fullest potential - yielding potent and effective treatment strategies with as little toxicity as possible - there must exist a means to discern the precise nature of the dysfunction in a given signaling network. The cardinal step in reaching this overall goal is identifying the full suite of proteins contributing to the disease and the nature of their aberrant activities (whether they be hyperphosphorylated or overexpressed, for example), a formidable task, in which the RPMA technology has enormous potential.

Nevertheless, the ability to translate this compendium of information into a diagnosis of network dysfunction is dependent on a detailed, a priori knowledge of the architecture of relevant signaling pathways, the kinetics of individual protein-protein interactions, as well as the functional structure of molecular control machinery such as feedback and feed-forward loops. Here, cell culture experiments, along with protein-binding studies, will form an essential backdrop for the tissue biopsy data through their ability to paint a dynamic, time-dependent portrait of a signaling network's response to stimulating ligands and/or other perturbations to cytoplasmic or nuclear signaling events.

Moreover, it is worth considering that these time-dependent studies are the experimental counterparts to the mathematical frameworks which assist both in determining kinetic parameters and elucidating network architectures, as well as providing a platform for in silico drug trials. Intracellular signaling is dynamic by its very nature, and the effects of any variations in stimulating signals should be understood in the context of a time-dependent study. Of course, after a period of unchanging conditions in the tissue microenvironment, intracellular protein concentrations may attain steady states in some cases, and in other cases, exhibit sustained oscillations about a "setpoint." On the other hand, should the extracellular milieu exhibit ongoing fluctuations in any properties that influence intracellular events, the processes involved in intracellular communication may constitute a very complex dynamic picture indeed.

Either way, an understanding of the relationship between the static picture of intracellular signaling furnished by the RPMA analysis of tissue biopsies and the dynamic picture furnished by corresponding cell culture studies and cognate mathematical models must emerge in order to assess the dysfunction of a particular tissue's signaling network, and thus, the true nature of a patient's disease. This reconciliation of paradigms is a complex question that must be addressed before significant advances can be made in this area of research.

Therefore, the goal of individualized therapy is to be pursued on a number of frontiers: high-throughput analysis of tissue biopsy data (via RPMA), the elucidation of the kinetics of intracellular signaling (via cell culture studies in combination with RPMA and mathematical modeling), and mathematical techniques to reconcile "static" tissue biopsy data with "dynamic" descriptions of intracellular signaling. Once the nature of signaling dysfunction is determined in an individual patient, the final frontier consists of identifying the most effective and least toxic strategy for launching an assault on the signaling network to elicit the desired clinical outcome. In the case of a particular cancer, this would usually mean tipping the balance of biochemical signaling events away from cell proliferation and cell survival and in favor of apoptosis.

\section{Combination Therapeutics: Insights from Mathematical Modeling}

Beyond the problem of identifying effective mechanisms to selectively modulate aberrantly activated signaling pathways with target-based drugs, a further challenge remains: toxicity. The narrow "therapeutic index" of much of the existing pharmacopoeia means that only a very restricted range of doses produces therapeutic benefit with tolerable toxicity, with doses outside of this range yielding either unacceptable toxicity or insufficient therapeutic benefit. Recent theoretical studies of intracellular signaling have focused on a new concept in the treatment of disease- " "network-targeted" combination therapywhich holds the promise of obviating many of these shortcomings. In this new approach, the emphasis is on distributing drug delivery among several targets, rather than concentrating the therapeutic intervention at a single signaling molecule. 
Combination therapies afford at least four key benefits in comparison with monotherapies:

1. For a given drug dose at each molecular target (or node), the attenuation of downstream signals is significantly enhanced when a multiplicity of targets is chosen rather than a single target, particularly when the nodes are serially linked (see Fig. 4) (Araujo et al. 2005).

2. The desired response may be produced with lower doses of the necessary agents when multiple nodes are targeted, rather than a single node in isolation (Araujo et al. 2004, 2005). Not only could this property reduce

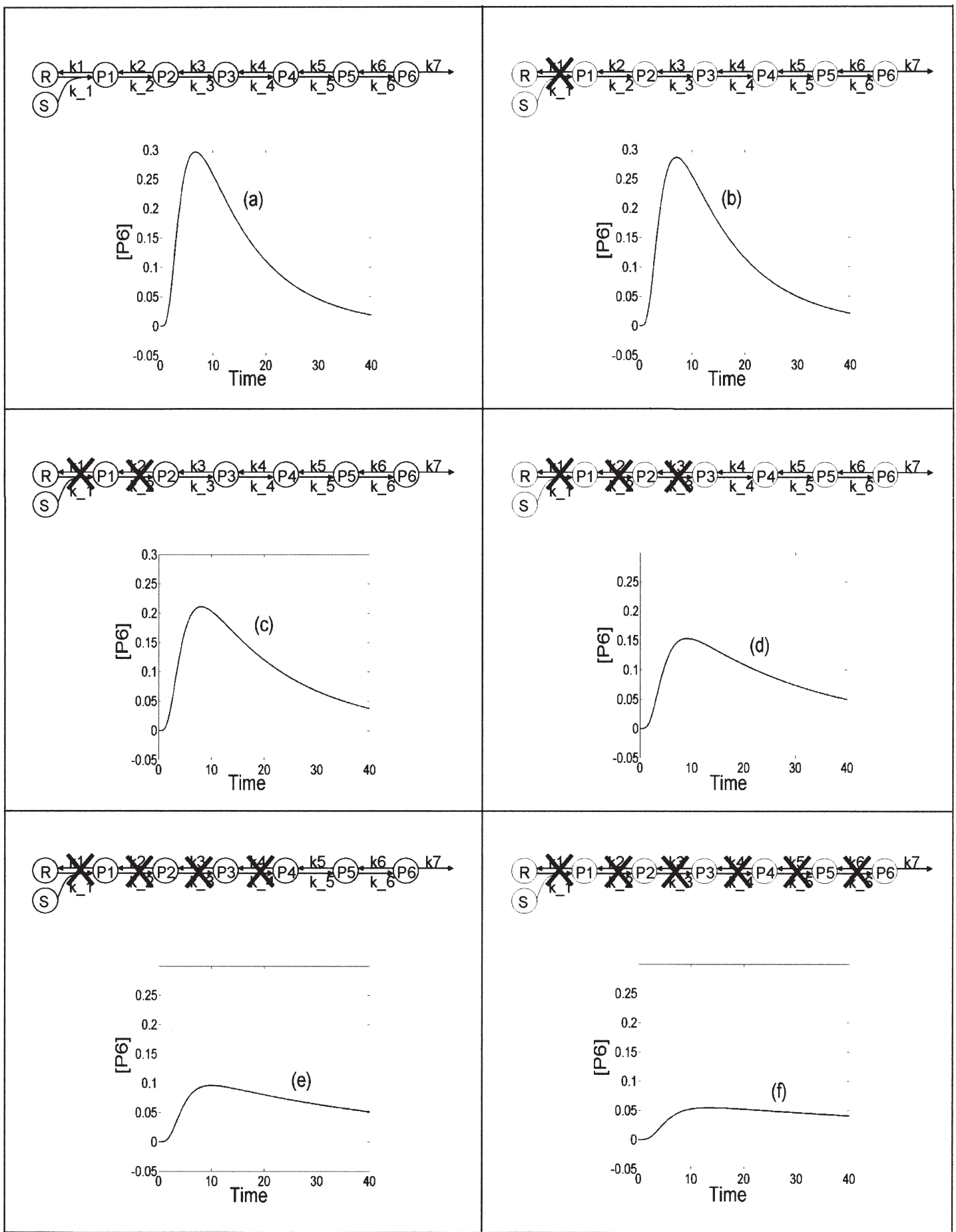

Figure 4. Mathematical modeling of the effects of combination therapy on signaling molecule cascades. The temporal evolution of a biochemical signal in a simple six-node network comprising proteins $\mathrm{P} 1$ through $\mathrm{P} 6$, in response to the binding of a stimulus, $\mathrm{S}$, to an upstream receptor, R. Case $a$ corresponds to an unperturbed network (no drugs). In case $b$, the receptor is targeted with a dose of $\mathrm{IC}_{50}$ of an appropriate inhibitor. In cases $c$ through $f$ a number of nodes are targeted, each with a dose of $\mathrm{IC}_{50}$. As more nodes are added to the treatment program, the output signal progressively diminishes. (Note that the biochemical signal under consideration is the concentration of the most downstream protein, P6.) (Adapted from Araujo et al. 2004.) 
the harmful side effects of drugs in current use, but it may impart clinical applicability to a huge compendium of agents which, of themselves, are too toxic at their therapeutically effective doses. This new concept may therefore spawn an enormous new repertoire of molecular-targeted therapeutics for clinical evaluation.

3. The location of target nodes in relation to the local architecture of the signaling network has important implications for the effectiveness of a therapy. Nodes embedded in negative feedback loops may represent very poor targets in some cases (Sauro and Kholodenko 2004), for example, since the automatic control characteristics of this signaling motif have the tendency to constrain the system response to follow a defined set point and resist the effects of any disturbances. Moreover, recent theoretical studies (Araujo et al. 2004) provide circumstantial evidence that receptors, being at the most upstream location in a signaling cascade, may represent quite poor targets. This is an important consideration in view of the number of drugs developed to target receptors (Iressa, Herceptin, Gleevec, etc.). On the other hand, receptors may represent a less complex therapeutic target in comparison with downstream nodes embedded in more complicated network architectures involving inter-pathway cross talk and feedback loops.

4. One of the consequences of the markedly nonlinear relationships between kinetic parameters and concentrations of signaling proteins is the nonadditive attenuation of signals for a multiplicity of target nodes, in comparison with targeting the same nodes individually (Araujo et al. 2005). Signal attenuation may therefore be synergistic, producing an extra inhibition that is not due to an extra dose of drug. We may therefore view this bonus inhibition as entirely nontoxic.

Thus, combination therapeutics, when conducted under the umbrella of an individualized assessment of intracellular signaling dysfunction, should allow much more effective disruption of biochemical signals and better control over overall cell behavior, with much lower drug doses and reduced systemic toxicity.

\section{CONCLUSION}

The promise of the integrated coupling of diagnosis with therapeutic intervention represents a new paradigm and will show benefit at multiple points in the biomedical network. For the biomedical researcher, the capability to profile the molecular content of diseased tissue will reveal a more complete understanding of disease pathophysiology. Pathologists will provide an entirely new service by including molecular pathway assessments to accompany their morphological descriptions. For clinicians, a more personalized, detailed molecular understanding of their patient's disease will provide more insight for developing therapeutic plans. Pharmaceutical company drug discovery teams will be able to perform an early assessment of a drug's effectiveness at a signal network level for a given molecular defect. This would provide a cost-effective means for prioritization of highvalue therapeutics early in the discovery and development process. Most importantly, patients will benefit through receiving a unique molecular characterization, resulting in a therapeutic plan that best fits their pathophysiology.

\section{REFERENCES}

Araujo R.P., Liotta L.A., and Petricoin E.F. 2004. Network-targeted combination therapy: A new concept in cancer treatment. Drug Discov Today: Therapeutic Strategies 1: 425.

Araujo R.P., Petricoin E.F., and Liotta L.A. 2005. A mathematical model of combination therapy using the EGFR signaling network. Biosystems 80: 57.

Emmert-Buck M.R., Bonner R.F., Smith P.D., Chuaqui R.F., Zhuang Z., Goldstein S.R., Weiss R.A., and Liotta L.A. 1996. Laser capture microdissection. Science 274: 998.

Espina V., Petricoin E.F., Liotta L.A., and Geho D. 2004. Application of sector protein microarrays to clinical samples. Clin. Proteomics 1: 91.

Geho D., Lahar N., Gurnani P., Huebschman M., Herrmann P., Espina V., Shi A., Wulfkuhle J., Garner H., Petricoin E., III, Liotta L.A., and Rosenblatt K.P. 2005. Pegylated, streptavidin-conjugated quantum dots are effective detection elements for reverse-phase protein microarrays. Bioconjug. Chem. 16: 559.

Goldbeter A. and Koshland D.E., Jr. 1981. An amplified sensitivity arising from covalent modification in biological systems. Proc. Natl. Acad. Sci. 78: 6840.

Huebschman M.L., Schultz R.A., and Garner H.R. 2002. Characteristics and capabilities of the hyperspectral imaging microscope. IEEE Eng. Med. Biol. Mag. 21: 104.

Hunter T. 2000. Signaling—2000 and beyond. Cell 100: 113.

King G., Payne S., Walker F., and Murray G.I. 1997. A highly sensitive detection method for immunohistochemistry using biotinylated tyramine. J. Pathol. 183: 237.

Liotta L.A. and Kohn E.C. 2001. The microenvironment of the tumour-host interface. Nature 411: 375.

Liotta L.A., Espina V., Mehta A.I., Calvert V., Rosenblatt K., Geho D., Munson P.J., Young L., Wulfkuhle J., and Petricoin E.F., III. 2003. Protein microarrays: Meeting analytical challenges for clinical applications. Cancer Cell 3: 317.

MacBeath G. 2002. Protein microarrays and proteomics. Nat. Genet. (suppl.) 32: 526.

MacBeath G. and Schreiber S.L. 2000. Printing proteins as microarrays for high throughput function determination. Science 289: 1760.

Ornstein D.K., Gillespie J.W., Paweletz C.P., Duray P.H., Herring J., Vocke C.D., Topalian S.L., Bostwick D.G., Linehan W.M., Petricoin E.F., III, and Emmert-Buck M.R. 2000. Proteomic analysis of laser capture microdissected human prostate cancer and in vitro prostate cell lines. Electrophoresis 21: 2235.

Paweletz, C.P., L. Charboneau, V.E. Bichsel, N.L. Simone, T. Chen, J.W. Gillespie, M.R. Emmert-Buck, M.J. Roth, I.E. Petricoin, and L.A. Liotta. 2001. Reverse phase protein microarrays which capture disease progression show activation of prosurvival pathways at the cancer invasion front. Oncogene 20: 1981.

Sauro H.M. and Kholodenko B.N. 2004. Quantitative analysis of signaling networks. Prog. Biophys. Mol. Biol. 86: 5.

Schultz R.A., Nielsen T., Zavaleta J.R., Ruch R., Wyatt R., and Garner H.R. 2001. Hyperspectral imaging: A novel approach for microscopic analysis. Cytometry 43: 239.

Sheehan K.M., Calvert V.S., Kay E.W., Lu Y., Fishman D., Espina V., Aquino J., Speer R., Araujo R., Mills G.B., Liotta L.A., Petricoin E.F., III, and Wulfkuhle J.D. 2005. Use of reverse phase protein microarrays and reference standard development for molecular network analysis of metastatic ovarian carcinoma. Mol. Cell. Proteomics 4: 346.

Tyson J.J., Chen K.C., and Novak B. 2003. Sniffers, buzzers, toggles and blinkers: Dynamics of regulatory and signaling pathways in the cell. Curr. Opin. Cell Biol. 15: 221.

Winters M.E., Mehta A.I., Petricoin E.F., III, Kohn E.C., and Liotta L.A. 2005. Supra-additive growth inhibition by a celecoxib 
analogue and carboxyamido-triazole is primarily mediated through apoptosis. Cancer Res. 65: 3853.

Wulfkuhle J.D., Aquino J.A., Calvert V.S., Fishman D.A., Coukos G., Liotta L.A., and Petricoin E.F., III. 2003. Signal pathway profiling of ovarian cancer from human tissue specimens using reverse-phase protein microarrays. Proteomics 3: 2085.
Zha H., Raffeld M., Charboneau L., Pittaluga S., Kwak L.W., Petricoin E., III, Liotta L.A., and Jaffe E.S. 2004. Similarities of prosurvival signals in Bcl-2-positive and Bcl-2-negative follicular lymphomas identified by reverse phase protein microarray. Lab. Invest. 84: 235.

Zhu H. and Snyder M. 2001. Protein arrays and microarrays. Curr. Opin. Chem. Biol. 5: 40. 


\section{$\$_{\text {CSH\& }}^{\infty} \mathrm{Cold}$ Spring Harbor Symposia SYMPOSIA on Quantitative Biology}

\section{Modeling of Protein Signaling Networks in Clinical Proteomics}

D.H. GEHO, E.F. PETRICOIN, L.A. LIOTTA, et al.

Cold Spring Harb Symp Quant Biol 2005 70: 517-524

Access the most recent version at doi:10.1101/sqb.2005.70.022

References This article cites 23 articles, 5 of which can be accessed free at:

http://symposium.cshlp.org/content/70/517.full.html\#ref-list-1

License

Email Alerting Receive free email alerts when new articles cite this article - sign up in

Service the box at the top right corner of the article or click here.

To subscribe to Cold Spring Harbor Symposia on Quantitative Biology go to: http://symposium.cshlp.org/subscriptions 